\title{
A commentary on "Pessary fitting for pelvic organ prolapse: parameters associated with specific reasons for failure"
}

\author{
Ghazaleh Rostaminia ${ }^{1}$ (D)
}

Received: 4 January 2022 / Accepted: 22 January 2022 / Published online: 25 February 2022

(c) The International Urogynecological Association 2022

This prospective observational case-control study [1] was aimed at investigating the parameters associated with unsuccessful pessary fitting using transperineal pelvic floor ultrasound (TPU). Women with symptomatic pelvic organ prolapse (POP) choosing pessary treatment were included. All women underwent an interview, clinical examination, and 3D/4D TPU. Pessary fitting was considered unsuccessful if initial fitting was unsuccessful or, after one or more fitting trials, the woman was not satisfied with any pessary and a different treatment was chosen. Women in whom the fitting failed were asked which one of the following was the reason for fitting failure: dislodgment (defined as a pessary that did not stay in place because it fell down or was expelled), failure to relieve POP symptoms, pain/discomfort, increased/ de novo urinary incontinence, or other reasons. With respect to the pessary type, a ring pessary (without or with support) was always tried at first. The TPU was performed in a supine position after bladder emptying, hiatal area at rest (HArest), maximum pelvic floor contraction (HActx), and maximum Valsalva maneuver (HAval) were manually segmented at the plane of minimal hiatal dimensions. From these parameters, the following were derived: displacement in contraction (DISPL-ctx), and displacement in Valsalva (DISPL-Val), and HARP ratio (i.e., hiatal area to ring pessary ratio).

A total of 130 subjects were included. Levator hiatal area (HA) on maximum Valsalva divided by ring pessary size ("Valsalva HARP ratio") was a predictor of unsuccessful fitting (OR 3.00, 95\% CI 1.15-7.81, $p=0.025$ ), with an area under the ROC curve (AUC) of 0.62 (95\% CI $0.50-0.74$, $p=0.04)$. Predictors of pessary dislodgment were: complete avulsion (OR 24.20, 95\% CI 2.46-237.84, $p$ value 0.01 ) and
Valsalva HARP ratio (OR 2.94, 95\% CI 1.32-6.55, $p$ value 0.01 ) with an area under the ROC curve (AUC) of 0.92 (95\% CI $0.84-0.99, p=0.00$ ). No significant parameter was identified in the prediction of pain/discomfort. Solitary predominant posterior compartment $\mathrm{POP}$ was a predictor of failure to relieve POP symptoms (OR 20.00, 95\% CI 3.48-115.02, $p$ value 0.00 ; AUC $0.75,95 \%$ CI $0.53-0.98, p=0.03$ ).

In summary, this study showed that unsuccessful pessary fitting was associated with LAM avulsion and Valsalva HARP ratio, as well as failure to relieve POP symptoms with isolated predominant posterior compartment POP. When a woman chooses pessary treatment for POP, LAM avulsion can be assessed. If present, the higher risk of dislodgment should be discussed. To minimize the risk of dislodgment, the maximum ring pessary size the woman can be fitted with should be selected. Compared with surgery, the advantages of pessary use are the low rate of complications and immediate effectiveness. Successful initial pessary fitting will increase the chance of continued pessary treatment.

\section{Reference}

1. Manzini C, Huub van der Vaart C, van den Noort F, Grob ATM, Withagen MIJ. Pessary fitting for pelvic organ prolapse: parameters associated with specific reasons for failure. Int Urogynecol J. 2022;https://doi.org/10.1007/s00192-021-05053-w.

Publisher's note Springer Nature remains neutral with regard to jurisdictional claims in published maps and institutional affiliations.
Ghazaleh Rostaminia

ghazalerostaminia@yahoo.com

1 NorthShore University Health System, Urogynecology Department, University of Chicago Pritzker School of Medicine, Skokie, IL, USA 